\title{
Multiple Positive Solutions of Third-Order BVP with Advanced Arguments and Stieltjes Integral Conditions
}

\author{
Jian Chang, Jian-Ping Sun, and Ya-Hong Zhao \\ Department of Applied Mathematics, Lanzhou University of Technology, Lanzhou 730050, China \\ Correspondence should be addressed to Jian-Ping Sun; jpsun@lut.cn
}

Received 24 March 2016; Revised 26 June 2016; Accepted 5 July 2016

Academic Editor: Gennaro Infante

Copyright (C) 2016 Jian Chang et al. This is an open access article distributed under the Creative Commons Attribution License, which permits unrestricted use, distribution, and reproduction in any medium, provided the original work is properly cited.

\begin{abstract}
We consider the following third-order boundary value problem with advanced arguments and Stieltjes integral boundary conditions: $u^{\prime \prime \prime}(t)+f(t, u(\alpha(t)))=0, t \in(0,1), u(0)=\gamma u\left(\eta_{1}\right)+\lambda_{1}[u]$ and $u^{\prime \prime}(0)=0, u(1)=\beta u\left(\eta_{2}\right)+\lambda_{2}[u]$, where $0<\eta_{1}<\eta_{2}<1$, $0 \leq \gamma, \beta \leq 1, \alpha:[0,1] \rightarrow[0,1]$ is continuous, $\alpha(t) \geq t$ for $t \in[0,1]$, and $\alpha(t) \leq \eta_{2}$ for $t \in\left[\eta_{1}, \eta_{2}\right]$. Under some suitable conditions, by applying a fixed point theorem due to Avery and Peterson, we obtain the existence of multiple positive solutions to the above problem. An example is also included to illustrate the main results obtained.
\end{abstract}

\section{Introduction}

Third-order differential equations arise from a variety of different areas of applied mathematics and physics, for example, in the deflection of a curved beam having a constant or varying cross-section, a three-layer beam, electromagnetic waves or gravity driven flows, and so on [1].

Recently, third-order boundary value problems (BVPs for short) have received much attention from many authors; see [2-20] and the references therein. However, it is necessary to point out that all the unknown functions in the abovementioned papers do not depend on advanced arguments.

In 2012, Jankowski [21] studied the existence of multiple positive solutions to the BVP

$$
\begin{aligned}
u^{\prime \prime \prime}(t)+h(t) f(t, u(\alpha(t))) & =0, \quad t \in(0,1), \\
u(0) & =u^{\prime \prime}(0)=0, \\
u(1) & =\beta u(\eta)+\lambda[u],
\end{aligned}
$$

where the unknown function $u$ depended on an advanced argument $\alpha$ satisfying the following condition:

$\left(A_{0}\right) \alpha:[0,1] \rightarrow[0,1]$ was continuous and $\alpha(t) \geq t$ for $t \in[0,1]$. $\lambda$ denoted a linear functional on $C[0,1]$ given by

$$
\lambda[u]=\int_{0}^{1} u(t) d \Lambda(t)
$$

involving a Stieltjes integral with a suitable function $\Lambda$ of bounded variation. The measure $d \Lambda$ could be a signed one. The situation with a signed measure $d \Lambda$ was first discussed in $[22,23]$ for second-order differential equations; it was also discussed in $[24,25]$ for second-order impulsive differential equations.

Among the boundary conditions in (1), only $u(1)$ was related to $u(\eta)$ and a Stieltjes integral. When $u(0)$ was also related to $u(\eta)$ and the Stieltjes integral, the authors in $[26,27]$ obtained the existence and multiplicity of positive solutions to the BVP

$$
\begin{aligned}
u^{\prime \prime \prime}(t)+f(t, u(\alpha(t))) & =0, \quad t \in(0,1), \\
u(0) & =\gamma u(\eta)+\lambda[u], \\
u^{\prime \prime}(0) & =0, \\
u(1) & =\beta u(\eta)+\lambda[u],
\end{aligned}
$$

where $\lambda[u]$ was defined as in (2) and the condition $\left(A_{0}\right)$ was imposed on the advanced argument $\alpha$. The main tools used were the Guo-Krasnoselskii fixed point theorem $[28,29]$ and a fixed point theorem due to Avery and Peterson [30]. 
In this paper, we are concerned with the following thirdorder BVP with advanced arguments and Stieltjes integral boundary conditions:

$$
\begin{aligned}
u^{\prime \prime \prime}(t)+f(t, u(\alpha(t))) & =0, \quad t \in(0,1), \\
u(0) & =\gamma u\left(\eta_{1}\right)+\lambda_{1}[u], \\
u^{\prime \prime}(0) & =0, \\
u(1) & =\beta u\left(\eta_{2}\right)+\lambda_{2}[u],
\end{aligned}
$$

where

$$
\lambda_{i}[u]=\int_{0}^{1} u(t) d \Lambda_{i}(t), \quad i=1,2,
$$

here $\Lambda_{1}$ and $\Lambda_{2}$ are suitable functions of bounded variation. It is important to indicate that it is not assumed that $\lambda_{i}[u](i=$ $1,2)$ is positive to all positive $u$. Throughout this paper, we always assume that $0<\eta_{1}<\eta_{2}<1,0 \leq \gamma, \beta \leq 1, f$ : $[0,1] \times[0,+\infty) \rightarrow[0,+\infty)$ is continuous, and the advanced argument $\alpha$ satisfies the following condition:

$\left(A_{0}^{\prime}\right) \alpha:[0,1] \rightarrow[0,1]$ is continuous, $\alpha(t) \geq t$ for $t \in$ $[0,1]$ and $\alpha(t) \leq \eta_{2}$ for $t \in\left[\eta_{1}, \eta_{2}\right]$.

In order to obtain our main results, we need the following concepts and fixed point theorem [30].

Let $E$ be a real Banach space and let $K$ be a cone in $E$.

A map $\Theta$ is said to be a nonnegative continuous convex functional on $K$ if $\Theta: K \rightarrow[0, \infty)$ is continuous and $\Theta(t u+$ $(1-t) v) \leq t \Theta(u)+(1-t) \Theta(v)$ for all $u, v \in K$ and $t \in[0,1]$.

Similarly, a map $\Phi$ is said to be a nonnegative continuous concave functional on $K$ if $\Phi: K \rightarrow[0, \infty)$ is continuous and $\Phi(t u+(1-t) v) \geq t \Phi(u)+(1-t) \Phi(v)$ for all $u, v \in K$ and $t \in[0,1]$.

Let $\varphi$ and $\Theta$ be nonnegative continuous convex functionals on $K$, let $\Phi$ be a nonnegative continuous concave functional on $K$, and let $\Psi$ be a nonnegative continuous functional on $K$. For positive numbers $a, b, c, d$, we define the following sets:

$$
\begin{aligned}
& K(\varphi, d)=\{u \in K: \varphi(u)<d\}, \\
& K(\varphi, \Phi, b, d)=\{u \in K: b \leq \Phi(u), \varphi(u) \leq d\}, \\
& K(\varphi, \Theta, \Phi, b, c, d) \\
& \quad=\{u \in K: b \leq \Phi(u), \Theta(u) \leq c, \varphi(u) \leq d\}, \\
& R(\varphi, \Psi, a, d)=\{u \in K: a \leq \Psi(u), \varphi(u) \leq d\} .
\end{aligned}
$$

Theorem 1 (Avery and Peterson fixed point theorem). Let $E$ be a real Banach space and let $K$ be a cone in $E$. Let $\varphi$ and $\Theta$ be nonnegative continuous convex functionals on $K$, let $\Phi$ be a nonnegative continuous concave functional on $K$, and let $\Psi$ be a nonnegative continuous functional on $K$ satisfying $\Psi(k u) \leq$ $k \Psi(u)$ for $0 \leq k \leq 1$, such that, for some positive numbers $M$ and $d$,

$$
\begin{gathered}
\Phi(u) \leq \Psi(u), \\
\|u\| \leq M \varphi(u)
\end{gathered}
$$

for all $u \in \overline{K(\varphi, d)}$. Suppose $S: \overline{K(\varphi, d)} \rightarrow \overline{K(\varphi, d)}$ is completely continuous and there exist positive numbers $a, b, c$ with $a<b$, such that

(C1) $\{u \in K(\varphi, \Theta, \Phi, b, c, d): \Phi(u)>b\} \neq \phi$ and $\Phi(S u)>b$ for $u \in K(\varphi, \Theta, \Phi, b, c, d)$;

(C2) $\Phi(S u)>b$ for $u \in K(\varphi, \Phi, b, d)$ with $\Theta(S u)>c$;

(C3) $\theta \notin R(\varphi, \Psi, a, d)$ and $\Psi(S u)<a$ for $u \in$ $R(\varphi, \Psi, a, d)$ with $\Psi(u)=a$.

Then $S$ has at least three fixed points $u_{1}, u_{2}, u_{3} \in \overline{K(\varphi, d)}$, such that

$$
\begin{aligned}
b & <\Phi\left(u_{1}\right), \\
a & <\Psi\left(u_{2}\right) \quad \text { with } \Phi\left(u_{2}\right)<b, \\
\Psi\left(u_{3}\right) & <a .
\end{aligned}
$$

\section{Main Results}

For convenience, we denote

$$
\begin{aligned}
\Delta & =(1-\gamma)\left(1-\eta_{2} \beta\right)+(1-\beta) \eta_{1} \gamma, \\
k(t, s) & =\frac{1}{2} \begin{cases}(1-t)\left(t-s^{2}\right), & 0 \leq s \leq t \leq 1, \\
t(1-s)^{2}, & 0 \leq t \leq s \leq 1,\end{cases} \\
\rho_{i} & =\left(1-\eta_{2} \beta\right) \int_{0}^{1} d \Lambda_{i}(t)-(1-\beta) \int_{0}^{1} t d \Lambda_{i}(t), \\
\tau_{i} & =\eta_{1} \gamma \int_{0}^{1} d \Lambda_{i}(t)+(1-\gamma) \int_{0}^{1} t d \Lambda_{i}(t), \quad
\end{aligned}
$$

In the remainder of this paper, we always assume that $\Delta-$ $\rho_{1}>0, \Delta-\tau_{2}>0$, and $\left(\Delta-\rho_{1}\right)\left(\Delta-\tau_{2}\right)>\rho_{2} \tau_{1}$, and for $\Lambda_{i}(i=1,2)$, the following conditions are fulfilled:

$$
\begin{aligned}
\int_{0}^{1} d \Lambda_{i}(t) & \geq \int_{0}^{1} t d \Lambda_{i}(t) \geq 0 \\
\kappa_{i}(s) & =\int_{0}^{1} k(t, s) d \Lambda_{i}(t) \geq 0
\end{aligned}
$$

$$
s \in[0,1], i=1,2 .
$$

Then, $\rho_{i} \geq 0, \tau_{i} \geq 0(i=1,2)$ and $\Delta>0$.

Lemma 2 (see [21]). One has $0 \leq k(t, s) \leq(1 / 2)(1+s)(1-s)^{2}$, $(t, s) \in[0,1] \times[0,1]$.

Lemma 3. For any $y \in C[0,1]$, the $B V P$

$$
\begin{aligned}
u^{\prime \prime \prime}(t) & =-y(t), \quad t \in(0,1), \\
u(0) & =\gamma u\left(\eta_{1}\right)+\lambda_{1}[u], \\
u^{\prime \prime}(0) & =0 \\
u(1) & =\beta u\left(\eta_{2}\right)+\lambda_{2}[u]
\end{aligned}
$$


has the unique solution

$$
\begin{aligned}
u(t)= & \frac{1-\eta_{2} \beta-t(1-\beta)}{\Delta} \lambda_{1}[u] \\
& +\frac{\eta_{1} \gamma+t(1-\gamma)}{\Delta} \lambda_{2}[u] \\
& +\frac{\left(1-\eta_{2} \beta\right) \gamma-t \gamma(1-\beta)}{\Delta} \int_{0}^{1} k\left(\eta_{1}, s\right) y(s) d s \\
& +\frac{\eta_{1} \beta \gamma+t \beta(1-\gamma)}{\Delta} \int_{0}^{1} k\left(\eta_{2}, s\right) y(s) d s \\
& +\int_{0}^{1} k(t, s) y(s) d s, \quad t \in[0,1] .
\end{aligned}
$$

Proof. By integrating the differential equation in (82) three times from 0 to $t$ and using the boundary condition $u^{\prime \prime}(0)=$ 0 , we know that

$$
u(t)=u(0)+u^{\prime}(0) t-\frac{1}{2} \int_{0}^{t}(t-s)^{2} y(s) d s
$$

$$
t \in[0,1] \text {. }
$$

And so,

$$
u^{\prime}(0)=u(1)-u(0)+\frac{1}{2} \int_{0}^{1}(1-s)^{2} y(s) d s
$$

In view of (13), (14), and the boundary conditions $u(0)=$ $\gamma u\left(\eta_{1}\right)+\lambda_{1}[u]$ and $u(1)=\beta u\left(\eta_{2}\right)+\lambda_{2}[u]$, we have

$$
\begin{aligned}
u(t)= & (1-t) \gamma u\left(\eta_{1}\right)+t \beta u\left(\eta_{2}\right)+(1-t) \lambda_{1}[u] \\
& +t \lambda_{2}[u]+\int_{0}^{1} k(t, s) y(s) d s, \quad t \in[0,1]
\end{aligned}
$$

Therefore,

$$
\begin{aligned}
u\left(\eta_{1}\right)= & \frac{1-\eta_{1}+\eta_{1} \beta-\eta_{2} \beta}{\Delta} \lambda_{1}[u]+\frac{\eta_{1}}{\Delta} \lambda_{2}[u] \\
& +\frac{1-\eta_{2} \beta}{\Delta} \int_{0}^{1} k\left(\eta_{1}, s\right) y(s) d s \\
& +\frac{\eta_{1} \beta}{\Delta} \int_{0}^{1} k\left(\eta_{2}, s\right) y(s) d s,
\end{aligned}
$$

$$
\begin{aligned}
u\left(\eta_{2}\right)= & \frac{1-\eta_{2}}{\Delta} \lambda_{1}[u]+\frac{\eta_{2}+\eta_{1} \gamma-\eta_{2} \gamma}{\Delta} \lambda_{2}[u] \\
& +\frac{\gamma-\eta_{2} \gamma}{\Delta} \int_{0}^{1} k\left(\eta_{1}, s\right) y(s) d s \\
& +\frac{1-\gamma+\eta_{1} \gamma}{\Delta} \int_{0}^{1} k\left(\eta_{2}, s\right) y(s) d s .
\end{aligned}
$$

Substituting (16) into (15), we get

$$
\begin{aligned}
u(t)= & \frac{1-\eta_{2} \beta-t(1-\beta)}{\Delta} \lambda_{1}[u] \\
& +\frac{\eta_{1} \gamma+t(1-\gamma)}{\Delta} \lambda_{2}[u] \\
& +\frac{\left(1-\eta_{2} \beta\right) \gamma-t \gamma(1-\beta)}{\Delta} \int_{0}^{1} k\left(\eta_{1}, s\right) y(s) d s \\
& +\frac{\eta_{1} \beta \gamma+t \beta(1-\gamma)}{\Delta} \int_{0}^{1} k\left(\eta_{2}, s\right) y(s) d s \\
& +\int_{0}^{1} k(t, s) y(s) d s, \quad t \in[0,1] .
\end{aligned}
$$

Let $C[0,1]$ be equipped with the maximum norm. Then $C[0,1]$ is a Banach space. If we let

$$
\begin{aligned}
K & =\left\{u \in C[0,1]: u(t) \geq 0, t \in[0,1], \min _{t \in\left[\eta_{1}, \eta_{2}\right]} u(t)\right. \\
& \left.\geq \Gamma\|u\|, \lambda_{i}[u] \geq 0, \quad i=1,2\right\},
\end{aligned}
$$

where

$$
\Gamma=\min \left\{\frac{\eta_{1}}{1-\gamma+\eta_{1} \gamma}, \frac{1-\eta_{2}}{1-\eta_{2} \beta}\right\},
$$

then $K$ is a cone in $C[0,1]$. Now, we define operators $T$ and $S$ on $K$ by

$$
\begin{aligned}
& (T u)(t)=\frac{1-\eta_{2} \beta-t(1-\beta)}{\Delta} \lambda_{1}[u]+\frac{\eta_{1} \gamma+t(1-\gamma)}{\Delta} \\
& \cdot \lambda_{2}[u]+(F u)(t), \quad t \in[0,1]
\end{aligned}
$$

$(\mathrm{Su})(t)$

$$
\begin{aligned}
& =\frac{\left(1-\eta_{2} \beta-t(1-\beta)\right)\left(\Delta-\tau_{2}\right)+\left(\eta_{1} \gamma+t(1-\gamma)\right) \rho_{2}}{\left(\Delta-\rho_{1}\right)\left(\Delta-\tau_{2}\right)-\rho_{2} \tau_{1}} \\
& \cdot \lambda_{1}[F u] \\
& +\frac{\left(1-\eta_{2} \beta-t(1-\beta)\right) \tau_{1}+\left(\eta_{1} \gamma+t(1-\gamma)\right)\left(\Delta-\rho_{1}\right)}{\left(\Delta-\rho_{1}\right)\left(\Delta-\tau_{2}\right)-\rho_{2} \tau_{1}} \\
& \cdot \lambda_{2}[F u]+(F u)(t), \quad t \in[0,1],
\end{aligned}
$$


where

$$
\begin{aligned}
(F u)(t)= & \frac{\left(1-\eta_{2} \beta\right) \gamma-t \gamma(1-\beta)}{\Delta} \\
& \cdot \int_{0}^{1} k\left(\eta_{1}, s\right) f(s, u(\alpha(s))) d s \\
& +\frac{\eta_{1} \beta \gamma+t \beta(1-\gamma)}{\Delta} \\
& \cdot \int_{0}^{1} k\left(\eta_{2}, s\right) f(s, u(\alpha(s))) d s \\
& +\int_{0}^{1} k(t, s) f(s, u(\alpha(s))) d s, \quad t \in[0,1] .
\end{aligned}
$$

Lemma 4. $T, S: K \rightarrow K$.

Proof. Let $u \in K$. Then it is easy to know that

$$
(T u)^{\prime \prime}(t)=-\int_{0}^{1} f(s, u(\alpha(s))) d s \leq 0, \quad t \in[0,1],
$$

which shows that $T u$ is concave down on $[0,1]$. In view of

$$
\begin{aligned}
& (F u)(0) \\
& =\frac{\left(1-\eta_{2} \beta\right) \gamma}{\Delta} \int_{0}^{1} k\left(\eta_{1}, s\right) f(s, u(\alpha(s))) d s \\
& \quad+\frac{\eta_{1} \beta \gamma}{\Delta} \int_{0}^{1} k\left(\eta_{2}, s\right) f(s, u(\alpha(s))) d s \geq 0,
\end{aligned}
$$

$(F u)(1)$

$$
\begin{aligned}
= & \frac{\left(1-\eta_{2}\right) \beta \gamma}{\Delta} \int_{0}^{1} k\left(\eta_{1}, s\right) f(s, u(\alpha(s))) d s \\
& +\frac{\left(1-\gamma+\eta_{1} \gamma\right) \beta}{\Delta} \int_{0}^{1} k\left(\eta_{2}, s\right) f(s, u(\alpha(s))) d s
\end{aligned}
$$$$
\geq 0 \text {, }
$$

we have

$$
\begin{aligned}
(\mathrm{Tu})(0)= & \frac{1-\eta_{2} \beta}{\Delta} \lambda_{1}[u]+\frac{\eta_{1} \gamma}{\Delta} \lambda_{2}[u]+(F u)(0) \\
\geq & 0, \\
(\mathrm{Tu})(1)= & \frac{\left(1-\eta_{2}\right) \beta}{\Delta} \lambda_{1}[u]+\frac{1-\gamma+\eta_{1} \gamma}{\Delta} \lambda_{2}[u] \\
& +(F u)(1) \geq 0 .
\end{aligned}
$$

So, $(T u)(t) \geq 0, t \in[0,1]$.

Now, we prove that $\min _{t \in\left[\eta_{1}, \eta_{2}\right]}(T u)(t) \geq \Gamma\|T u\|$. To do it we consider two cases.
Case 1. Let $(T u)\left(\eta_{1}\right) \leq(T u)\left(\eta_{2}\right)$. Then $\min _{t \in\left[\eta_{1}, \eta_{2}\right]}(T u)(t)=$ $(T u)\left(\eta_{1}\right)$ and there exists $\bar{t} \in\left[\eta_{1}, 1\right]$ such that $\|T u\|=(T u)(\bar{t})$. If $\bar{t} \in\left[\eta_{1}, \eta_{2}\right]$, then

$$
\frac{(T u)(\bar{t})-(T u)(0)}{\bar{t}} \leq \frac{(T u)\left(\eta_{1}\right)-(T u)(0)}{\eta_{1}} .
$$

So,

$$
\|T u\| \leq \frac{\eta_{2}}{\eta_{1}}(T u)\left(\eta_{1}\right)-\frac{\eta_{2}-\eta_{1}}{\eta_{1}}(T u)(0),
$$

which together with

$$
(T u)(0)=\gamma(T u)\left(\eta_{1}\right)+\lambda_{1}[u]
$$

implies that

$$
\|T u\| \leq \frac{\eta_{2}-\left(\eta_{2}-\eta_{1}\right) \gamma}{\eta_{1}}(T u)\left(\eta_{1}\right)
$$

that is,

$$
\min _{t \in\left[\eta_{1}, \eta_{2}\right]}(T u)(t) \geq \frac{\eta_{1}}{\eta_{2}-\left(\eta_{2}-\eta_{1}\right) \gamma}\|T u\|
$$

If $\bar{t} \in\left(\eta_{2}, 1\right]$, then

$$
\frac{(T u)(\bar{t})-(T u)\left(\eta_{1}\right)}{\bar{t}-\eta_{1}} \leq \frac{(T u)\left(\eta_{2}\right)-(T u)\left(\eta_{1}\right)}{\eta_{2}-\eta_{1}} .
$$

So,

$$
\|T u\| \leq \frac{1-\eta_{1}}{\eta_{2}-\eta_{1}}(T u)\left(\eta_{2}\right)-\frac{1-\eta_{2}}{\eta_{2}-\eta_{1}}(T u)\left(\eta_{1}\right) .
$$

On the other hand, it follows from

$$
\frac{(T u)\left(\eta_{1}\right)-(T u)(0)}{\eta_{1}} \geq \frac{(T u)\left(\eta_{2}\right)-(T u)(0)}{\eta_{2}}
$$

and (27) that

$$
(\mathrm{Tu})\left(\eta_{2}\right) \leq \frac{\eta_{2}-\left(\eta_{2}-\eta_{1}\right) \gamma}{\eta_{1}}(\mathrm{Tu})\left(\eta_{1}\right),
$$

which together with (31) implies that

$$
\|T u\| \leq \frac{1-\gamma+\eta_{1} \gamma}{\eta_{1}}(T u)\left(\eta_{1}\right) ;
$$

that is,

$$
\min _{t \in\left[\eta_{1}, \eta_{2}\right]}(T u)(t) \geq \frac{\eta_{1}}{1-\gamma+\eta_{1} \gamma}\|T u\| .
$$


Case 2. Let $(T u)\left(\eta_{1}\right)>(T u)\left(\eta_{2}\right)$. Then $\min _{t \in\left[\eta_{1}, \eta_{2}\right]}(T u)(t)=$ $(T u)\left(\eta_{2}\right)$ and there exists $\bar{t} \in\left[0, \eta_{2}\right)$ such that $\|T u\|=(T u)(\bar{t})$.

If $\bar{t} \in\left[0, \eta_{1}\right]$, then

$$
\frac{(T u)\left(\eta_{2}\right)-(T u)(\bar{t})}{\eta_{2}-\bar{t}} \geq \frac{(T u)\left(\eta_{2}\right)-(T u)\left(\eta_{1}\right)}{\eta_{2}-\eta_{1}}
$$

So,

$$
\|T u\| \leq \frac{\eta_{2}}{\eta_{2}-\eta_{1}}(T u)\left(\eta_{1}\right)-\frac{\eta_{1}}{\eta_{2}-\eta_{1}}(T u)\left(\eta_{2}\right) .
$$

At the same time, since

$$
\frac{(T u)\left(\eta_{2}\right)-(T u)\left(\eta_{1}\right)}{\eta_{2}-\eta_{1}} \geq \frac{(T u)(1)-(T u)\left(\eta_{1}\right)}{1-\eta_{1}}
$$

we have

$$
(T u)\left(\eta_{1}\right) \leq \frac{1-\eta_{1}}{1-\eta_{2}}(T u)\left(\eta_{2}\right)-\frac{\eta_{2}-\eta_{1}}{1-\eta_{2}}(T u)(1),
$$

which together with

$$
(T u)(1)=\beta(T u)\left(\eta_{2}\right)+\lambda_{2}[u]
$$

implies that

$$
(T u)\left(\eta_{1}\right) \leq \frac{1-\eta_{1}-\left(\eta_{2}-\eta_{1}\right) \beta}{1-\eta_{2}}(T u)\left(\eta_{2}\right) .
$$

In view of (37) and (41), we have

$$
\|T u\| \leq \frac{1-\eta_{2} \beta}{1-\eta_{2}}(T u)\left(\eta_{2}\right) ;
$$

that is,

$$
\min _{t \in\left[\eta_{1}, \eta_{2}\right]}(T u)(t) \geq \frac{1-\eta_{2}}{1-\eta_{2} \beta}\|T u\|
$$

If $\bar{t} \in\left(\eta_{1}, \eta_{2}\right)$, then

$$
\frac{(T u)(1)-(T u)(\bar{t})}{1-\bar{t}} \geq \frac{(T u)(1)-(T u)\left(\eta_{2}\right)}{1-\eta_{2}}
$$

So,

$$
\|T u\| \leq \frac{1-\eta_{1}}{1-\eta_{2}}(T u)\left(\eta_{2}\right)-\frac{\eta_{2}-\eta_{1}}{1-\eta_{2}}(T u)(1),
$$

which together with (40) implies that

$$
\|T u\| \leq \frac{1-\eta_{1}-\left(\eta_{2}-\eta_{1}\right) \beta}{1-\eta_{2}}(T u)\left(\eta_{2}\right)
$$

that is,

$$
\min _{t \in\left[\eta_{1}, \eta_{2}\right]}(T u)(t) \geq \frac{1-\eta_{2}}{1-\eta_{1}-\left(\eta_{2}-\eta_{1}\right) \beta}\|T u\| .
$$

It follows from (29), (35), (43), and (47) that

$$
\min _{t \in\left[\eta_{1}, \eta_{2}\right]}(T u)(t) \geq \Gamma\|T u\|
$$

Finally, we need to show that $\lambda_{i}[T u] \geq 0, i=1,2$. Since

$$
\lambda_{i}[F u]=\int_{0}^{1} \frac{\left(1-\eta_{2} \beta\right) \gamma-t \gamma(1-\beta)}{\Delta}
$$

$$
\begin{aligned}
& \cdot \int_{0}^{1} k\left(\eta_{1}, s\right) f(s, u(\alpha(s))) d s d \Lambda_{i}(t) \\
& +\int_{0}^{1} \frac{\eta_{1} \beta \gamma+t \beta(1-\gamma)}{\Delta} \\
& \cdot \int_{0}^{1} k\left(\eta_{2}, s\right) f(s, u(\alpha(s))) d s d \Lambda_{i}(t) \\
& +\int_{0}^{1} \int_{0}^{1} k(t, s) f(s, u(\alpha(s))) d s d \Lambda_{i}(t)=\frac{\gamma \rho_{i}}{\Delta}
\end{aligned}
$$

$$
\begin{aligned}
& \cdot \int_{0}^{1} k\left(\eta_{1}, s\right) f(s, u(\alpha(s))) d s+\frac{\beta \tau_{i}}{\Delta} \\
& \cdot \int_{0}^{1} k\left(\eta_{2}, s\right) f(s, u(\alpha(s))) d s
\end{aligned}
$$

$$
+\int_{0}^{1} \kappa_{i}(s) f(s, u(\alpha(s))) d s \geq 0, \quad i=1,2,
$$

we have

$$
\begin{aligned}
& \lambda_{i}[T u]=\frac{\rho_{i}}{\Delta} \lambda_{1}[u]+\frac{\tau_{i}}{\Delta} \lambda_{2}[u]+\lambda_{i}[F u] \geq 0, \\
& i=1,2 \text {. }
\end{aligned}
$$

Therefore, $T: K \rightarrow K$. Similarly, we may prove that $S:$ $K \rightarrow K$.

Lemma 5. T and S have the same fixed points in $K$. 
Proof. On the one hand, if $u \in K$ is a fixed point of $S$, that is, $u=S u$, then

$$
\begin{aligned}
\lambda_{1}[u] & =\lambda_{1}[S u] \\
= & \int_{0}^{1}\left(\frac{\left(1-\eta_{2} \beta-t(1-\beta)\right)\left(\Delta-\tau_{2}\right)+\left(\eta_{1} \gamma+t(1-\gamma)\right) \rho_{2}}{\left(\Delta-\rho_{1}\right)\left(\Delta-\tau_{2}\right)-\rho_{2} \tau_{1}}\right. \\
& \cdot \lambda_{1}[F u] \\
& +\frac{\left(1-\eta_{2} \beta-t(1-\beta)\right) \tau_{1}+\left(\eta_{1} \gamma+t(1-\gamma)\right)\left(\Delta-\rho_{1}\right)}{\left(\Delta-\rho_{1}\right)\left(\Delta-\tau_{2}\right)-\rho_{2} \tau_{1}} \\
& \left.\cdot \lambda_{2}[F u]+(F u)(t)\right) d \Lambda_{1}(t) \\
& =\frac{\Delta\left(\Delta-\tau_{2}\right) \lambda_{1}[F u]+\Delta \tau_{1} \lambda_{2}[F u]}{\left(\Delta-\rho_{1}\right)\left(\Delta-\tau_{2}\right)-\rho_{2} \tau_{1}}, \\
\lambda_{2} & {[u]=\lambda_{2}[S u] } \\
& =\int_{0}^{1}\left(\frac{\left(1-\eta_{2} \beta-t(1-\beta)\right)\left(\Delta-\tau_{2}\right)+\left(\eta_{1} \gamma+t(1-\gamma)\right) \rho_{2}}{\left(\Delta-\rho_{1}\right)\left(\Delta-\tau_{2}\right)-\rho_{2} \tau_{1}}\right. \\
& \cdot \lambda_{1}[F u] \\
& +\frac{\Delta \rho_{2} \lambda_{1}[F u]+\Delta\left(\Delta-\rho_{1}\right) \lambda_{2}[F u]}{\left(\Delta-\rho_{1}\right)\left(\Delta-\tau_{2}\right)-\rho_{2} \tau_{1}}, \\
& \left.\lambda_{2}[F u]+(F u)(t)\right) d \Lambda_{2}(t) \\
& \frac{\left(1-\eta_{2} \beta-t(1-\beta)\right) \tau_{1}+\left(\eta_{1} \gamma+t(1-\gamma)\right)\left(\Delta-\rho_{1}\right)}{\left(\Delta-\tau_{2}\right)-\rho_{2} \tau_{1}} \\
&
\end{aligned}
$$

which shows that

$$
\begin{aligned}
& \lambda_{1}[F u]=\frac{\left(\Delta-\rho_{1}\right) \lambda_{1}[u]-\tau_{1} \lambda_{2}[u]}{\Delta}, \\
& \lambda_{2}[F u]=\frac{\left(\Delta-\tau_{2}\right) \lambda_{2}[u]-\rho_{2} \lambda_{1}[u]}{\Delta} .
\end{aligned}
$$

So,

$$
\begin{aligned}
u(t) & =(S u)(t) \\
& =\frac{\left(1-\eta_{2} \beta-t(1-\beta)\right)\left(\Delta-\tau_{2}\right)+\left(\eta_{1} \gamma+t(1-\gamma)\right) \rho_{2}}{\left(\Delta-\rho_{1}\right)\left(\Delta-\tau_{2}\right)-\rho_{2} \tau_{1}} \\
& \cdot \lambda_{1}[F u] \\
& +\frac{\left(1-\eta_{2} \beta-t(1-\beta)\right) \tau_{1}+\left(\eta_{1} \gamma+t(1-\gamma)\right)\left(\Delta-\rho_{1}\right)}{\left(\Delta-\rho_{1}\right)\left(\Delta-\tau_{2}\right)-\rho_{2} \tau_{1}} \\
& \cdot \lambda_{2}[F u]+(F u)(t)=\frac{1-\eta_{2} \beta-t(1-\beta)}{\Delta} \lambda_{1}[u] \\
& +\frac{\eta_{1} \gamma+t(1-\gamma)}{\Delta} \lambda_{2}[u]+(F u)(t)=(T u)(t),
\end{aligned}
$$$$
t \in[0,1],
$$

which indicates that $u$ is a fixed point of $T$.
On the other hand, if $u \in K$ is a fixed point of $T$, that is, $u=T u$, then

$$
\begin{gathered}
\lambda_{i}[u]=\lambda_{i}[T u]=\int_{0}^{1}\left(\frac{1-\eta_{2} \beta-t(1-\beta)}{\Delta} \lambda_{1}[u]\right. \\
\left.+\frac{\eta_{1} \gamma+t(1-\gamma)}{\Delta} \lambda_{2}[u]+(F u)(t)\right) d \Lambda_{i}(t) \\
=\frac{\rho_{i}}{\Delta} \lambda_{1}[u]+\frac{\tau_{i}}{\Delta} \lambda_{2}[u]+\lambda_{i}[F u], \quad i=1,2
\end{gathered}
$$

which shows that

$$
\begin{aligned}
& \lambda_{1}[u]=\frac{\Delta\left(\Delta-\tau_{2}\right) \lambda_{1}[F u]+\Delta \tau_{1} \lambda_{2}[F u]}{\left(\Delta-\rho_{1}\right)\left(\Delta-\tau_{2}\right)-\rho_{2} \tau_{1}}, \\
& \lambda_{2}[u]=\frac{\Delta \rho_{2} \lambda_{1}[F u]+\Delta\left(\Delta-\rho_{1}\right) \lambda_{2}[F u]}{\left(\Delta-\rho_{1}\right)\left(\Delta-\tau_{2}\right)-\rho_{2} \tau_{1}} .
\end{aligned}
$$

So,

$$
\begin{aligned}
u(t) & =(T u)(t)=\frac{1-\eta_{2} \beta-t(1-\beta)}{\Delta} \lambda_{1}[u] \\
+ & \frac{\eta_{1} \gamma+t(1-\gamma)}{\Delta} \lambda_{2}[u]+(F u)(t) \\
& =\frac{\left(1-\eta_{2} \beta-t(1-\beta)\right)\left(\Delta-\tau_{2}\right)+\left(\eta_{1} \gamma+t(1-\gamma)\right) \rho_{2}}{\left(\Delta-\rho_{1}\right)\left(\Delta-\tau_{2}\right)-\rho_{2} \tau_{1}} \\
\cdot & \lambda_{1}[F u] \quad \\
+ & \frac{\left(1-\eta_{2} \beta-t(1-\beta)\right) \tau_{1}+\left(\eta_{1} \gamma+t(1-\gamma)\right)\left(\Delta-\rho_{1}\right)}{\left(\Delta-\rho_{1}\right)\left(\Delta-\tau_{2}\right)-\rho_{2} \tau_{1}} \\
\cdot & \lambda_{2}[F u]+(F u)(t)=(S u)(t), \quad t \in[0,1],
\end{aligned}
$$

which indicates that $u$ is a fixed point of $S$.

Lemma 6. $T, S: K \rightarrow K$ is completely continuous.

Proof. First, by Lemma 4, we know that $T(K) \subset K$.

Next, we show that $T$ is compact.

Let $D \subset K$ be a bounded set. Then there exists $M_{1}>0$ such that $\|u\| \leq M_{1}$ for any $u \in D$. Since $\Lambda_{1}$ and $\Lambda_{2}$ are functions of bounded variation, there exists $M_{2}>0$ such that

$$
\sum_{j=1}^{n}\left|\Lambda_{i}\left(t_{j}\right)-\Lambda_{i}\left(t_{j-1}\right)\right| \leq M_{2}, \quad i=1,2
$$

for any partition $\Delta^{\prime}: 0=t_{0}<t_{1}<\cdots<t_{n-1}<t_{n}=1$. Let

$$
M_{3}=\sup \left\{f(t, u):(t, u) \in[0,1] \times\left[0, M_{1}\right]\right\} .
$$


Then for any $u \in D$, we have

$$
\begin{aligned}
\|T u\| & =\max _{t \in[0,1]}(T u)(t) \\
\leq & \frac{1-\eta_{2} \beta}{\Delta} \lambda_{1}[u]+\frac{1-\gamma+\eta_{1} \gamma}{\Delta} \lambda_{2}[u] \\
& +\frac{\left(1-\eta_{2} \beta\right) \gamma}{\Delta} \int_{0}^{1} k\left(\eta_{1}, s\right) f(s, u(\alpha(s))) d s \\
& +\frac{\left(1-\gamma+\eta_{1} \gamma\right) \beta}{\Delta} \int_{0}^{1} k\left(\eta_{2}, s\right) f(s, u(\alpha(s))) d s \\
& +\frac{1}{2} \int_{0}^{1}(1+s)(1-s)^{2} f(s, u(\alpha(s))) d s \\
\leq & \frac{1-\eta_{2} \beta}{\Delta} M_{1} M_{2}+\frac{1-\gamma+\eta_{1} \gamma}{\Delta} M_{1} M_{2} \\
& +\frac{\left(1-\eta_{2} \beta\right) \gamma M_{3}}{\Delta} \int_{0}^{1} k\left(\eta_{1}, s\right) d s \\
& +\frac{\left(1-\gamma+\eta_{1} \gamma\right) \beta M_{3}}{\Delta} \int_{0}^{1} k\left(\eta_{2}, s\right) d s+\frac{5}{24} M_{3},
\end{aligned}
$$

which shows that $T(D)$ is uniformly bounded.

On the other hand, for any $\varepsilon>0$, since $k(t, s)$ is uniformly continuous on $[0,1] \times[0,1]$, there exists $\delta_{1}(\varepsilon)>0$ such that, for any $t_{1}, t_{2} \in[0,1]$ with $\left|t_{1}-t_{2}\right|<\delta_{1}(\varepsilon)$,

$$
\left|k\left(t_{1}, s\right)-k\left(t_{2}, s\right)\right|<\frac{\varepsilon}{5\left(M_{3}+1\right)}, \quad s \in[0,1] .
$$

Let

$$
\begin{aligned}
\delta= & \min \left\{\delta_{1}(\varepsilon), \frac{\varepsilon \Delta}{5\left((1-\beta) M_{1} M_{2}+1\right)},\right. \\
& \frac{\varepsilon \Delta}{5\left((1-\gamma) M_{1} M_{2}+1\right)}, \\
& \frac{\varepsilon \Delta}{5\left((1-\beta) \gamma M_{3} \int_{0}^{1} k\left(\eta_{1}, s\right) d s+1\right)}, \\
& \left.\frac{\varepsilon \Delta}{5\left((1-\gamma) \beta M_{3} \int_{0}^{1} k\left(\eta_{2}, s\right) d s+1\right)}\right\} .
\end{aligned}
$$

Then for any $u \in D, t_{1}, t_{2} \in[0,1]$ with $\left|t_{1}-t_{2}\right|<\delta$, we have

$$
\begin{aligned}
\left|(T u)\left(t_{1}\right)-(T u)\left(t_{2}\right)\right|=\mid \frac{\left(t_{1}-t_{2}\right)(\beta-1)}{\Delta} \lambda_{1}[u] \\
+\frac{\left(t_{1}-t_{2}\right)(1-\gamma)}{\Delta} \lambda_{2}[u]+\frac{\left(t_{1}-t_{2}\right)(\beta-1) \gamma}{\Delta} \\
\quad \cdot \int_{0}^{1} k\left(\eta_{1}, s\right) f(s, u(\alpha(s))) d s \\
+\frac{\left(t_{1}-t_{2}\right)(1-\gamma) \beta}{\Delta} \int_{0}^{1} k\left(\eta_{2}, s\right) f(s, u(\alpha(s))) d s \\
+\int_{0}^{1}\left(k\left(t_{1}, s\right)-k\left(t_{2}, s\right)\right) f(s, u(\alpha(s))) d s \mid \\
\quad \leq \frac{(1-\beta)\left|t_{1}-t_{2}\right|}{\Delta} \lambda_{1}[u]+\frac{(1-\gamma)\left|t_{1}-t_{2}\right|}{\Delta} \lambda_{2}[u] \\
\quad+\int_{0}^{1} k\left(\eta_{2}, s\right) d s+M_{3} \int_{0}^{1}\left|k\left(t_{1}, s\right)-k\left(t_{2}, s\right)\right| d s \\
+\frac{(1-\beta) \gamma\left|t_{1}-t_{2}\right|}{\Delta} \int_{0}^{1} k\left(\eta_{1}, s\right) f(s, u(\alpha(s))) d s \\
+\int_{0}^{1}\left|k\left(t_{1}, s\right)-k\left(t_{2}, s\right)\right| f(s, u(\alpha(s))) d s \\
+\frac{(1-\gamma)\left|t_{1}-t_{2}\right| M_{1} M_{2}}{\Delta} \\
\quad \frac{(1-\gamma)\left|t_{1}-t_{2}\right| M_{1} M_{2}}{\Delta}+\frac{(1-\beta) \gamma\left|t_{1}-t_{2}\right| M_{3}}{\Delta} \\
\quad \int_{0}^{1} k\left(\eta_{2}, s\right) f(s, u(\alpha(s))) d s
\end{aligned}
$$

which shows that $T(D)$ is equicontinuous. It follows from Arzela-Ascoli theorem that $T(D)$ is relatively compact. Thus, we have shown that $T$ is a compact operator.

Finally, we prove that $T$ is continuous.

Assume that $u_{n}, u \in K$ and $\lim _{n \rightarrow \infty} u_{n}=u$. Then there exists $M_{4}>0$ such that $\|u\| \leq M_{4}$ and $\left\|u_{n}\right\| \leq M_{4}, n=$ $1,2, \ldots$. For any $\varepsilon>0$, since $f(s, x)$ is uniformly continuous on $[0,1] \times\left[0, M_{4}\right]$, there exists $\delta>0$ such that, for any $x_{1}, x_{2} \in$ $\left[0, M_{4}\right]$ with $\left|x_{1}-x_{2}\right|<\delta$,

$$
\left|f\left(s, x_{1}\right)-f\left(s, x_{2}\right)\right|<\frac{\varepsilon}{\left(3\left(2-\eta_{2} \beta-\beta\right) \gamma / \Delta\right) \int_{0}^{1} k\left(\eta_{1}, s\right) d s+\left(3\left(1-\gamma+\eta_{1} \gamma\right) \beta / \Delta\right) \int_{0}^{1} k\left(\eta_{2}, s\right) d s+5 / 8}, \quad s \in[0,1] .
$$


At the same time, since $\lim _{n \rightarrow \infty} u_{n}=u$, there exists positive integer $N$ such that, for any $n>N$,

$$
\begin{gathered}
\left\|u_{n}-u\right\|<\min \left\{\delta, \frac{\varepsilon \Delta}{3\left(2-\eta_{2} \beta-\beta\right) M_{2}},\right. \\
\left.\frac{\varepsilon \Delta}{3\left(1-\gamma+\eta_{1} \gamma\right) M_{2}}\right\} .
\end{gathered}
$$

It follows from (63) and (64) that, for any $n>N$,

$$
\begin{aligned}
& \left\|T u_{n}-T u\right\|=\max _{t \in[0,1]}\left|\left(T u_{n}\right)(t)-(T u)(t)\right| \\
& \leq \frac{2-\eta_{2} \beta-\beta}{\Delta}\left|\lambda_{1}\left[u_{n}\right]-\lambda_{1}[u]\right| \\
& +\frac{1-\gamma+\eta_{1} \gamma}{\Delta}\left|\lambda_{2}\left[u_{n}\right]-\lambda_{2}[u]\right|+\frac{\left(2-\eta_{2} \beta-\beta\right) \gamma}{\Delta} \\
& \cdot \int_{0}^{1} k\left(\eta_{1}, s\right)\left|f\left(s, u_{n}(\alpha(s))\right)-f(s, u(\alpha(s)))\right| d s \\
& +\frac{\left(1-\gamma+\eta_{1} \gamma\right) \beta}{\Delta} \int_{0}^{1} k\left(\eta_{2}, s\right) \mid f\left(s, u_{n}(\alpha(s))\right) \\
& -f(s, u(\alpha(s))) \mid d s+\frac{1}{2} \int_{0}^{1}(1+s)(1-s)^{2} \\
& \cdot\left|f\left(s, u_{n}(\alpha(s))\right)-f(s, u(\alpha(s)))\right| d s \\
& \leq \frac{2-\eta_{2} \beta-\beta}{\Delta}\left\|u_{n}-u\right\| M_{2}+\frac{1-\gamma+\eta_{1} \gamma}{\Delta}\left\|u_{n}-u\right\| \\
& \cdot M_{2}+\int_{0}^{1}\left(\frac{\left(2-\eta_{2} \beta-\beta\right) \gamma}{\Delta} k\left(\eta_{1}, s\right)\right. \\
& \left.+\frac{\left(1-\gamma+\eta_{1} \gamma\right) \beta}{\Delta} k\left(\eta_{2}, s\right)+\frac{1}{2}(1+s)(1-s)^{2}\right) \\
& \cdot\left|f\left(s, u_{n}(\alpha(s))\right)-f(s, u(\alpha(s)))\right| d s<\varepsilon,
\end{aligned}
$$

which indicates that $T$ is continuous.

Therefore, $T: K \rightarrow K$ is completely continuous. Similarly, we can prove that $S: K \rightarrow K$ is also completely continuous.

For convenience, we denote

$$
\begin{aligned}
D_{1}= & \frac{\gamma \rho_{1}}{\Delta} \int_{0}^{1} k\left(\eta_{1}, s\right) d s+\frac{\beta \tau_{1}}{\Delta} \int_{0}^{1} k\left(\eta_{2}, s\right) d s \\
& +\int_{0}^{1} \kappa_{1}(s) d s, \\
D_{2}= & \frac{\gamma \rho_{2}}{\Delta} \int_{0}^{1} k\left(\eta_{1}, s\right) d s+\frac{\beta \tau_{2}}{\Delta} \int_{0}^{1} k\left(\eta_{2}, s\right) d s \\
& +\int_{0}^{1} \kappa_{2}(s) d s,
\end{aligned}
$$

$$
\begin{aligned}
D_{3}= & \frac{\left(1-\eta_{2} \beta\right) \gamma}{\Delta} \int_{0}^{1} k\left(\eta_{1}, s\right) d s \\
& +\frac{\left(1-\gamma+\eta_{1} \gamma\right) \beta}{\Delta} \int_{0}^{1} k\left(\eta_{2}, s\right) d s+\frac{5}{24}, \\
D_{4}= & \frac{\gamma \rho_{1}}{\Delta} \int_{\eta_{1}}^{\eta_{2}} k\left(\eta_{1}, s\right) d s+\frac{\beta \tau_{1}}{\Delta} \int_{\eta_{1}}^{\eta_{2}} k\left(\eta_{2}, s\right) d s \\
& +\int_{\eta_{1}}^{\eta_{2}} \kappa_{1}(s) d s, \\
D_{5}= & \frac{\gamma \rho_{2}}{\Delta} \int_{\eta_{1}}^{\eta_{2}} k\left(\eta_{1}, s\right) d s+\frac{\beta \tau_{2}}{\Delta} \int_{\eta_{1}}^{\eta_{2}} k\left(\eta_{2}, s\right) d s \\
& +\int_{\eta_{1}}^{\eta_{2}} \kappa_{2}(s) d s, \\
D_{6}= & \frac{\gamma\left(1-\eta_{2}\right)}{\Delta} \int_{\eta_{1}}^{\eta_{2}} k\left(\eta_{1}, s\right) d s \\
& +\frac{1-\gamma+\eta_{1} \gamma}{\Delta} \int_{\eta_{1}}^{\eta_{2}} k\left(\eta_{2}, s\right) d s .
\end{aligned}
$$

Let

$$
\begin{aligned}
\mu> & \frac{\left(1-\eta_{2} \beta\right)\left(\Delta-\tau_{2}\right)+\left(1-\gamma+\eta_{1} \gamma\right) \rho_{2}}{\left(\Delta-\rho_{1}\right)\left(\Delta-\tau_{2}\right)-\rho_{2} \tau_{1}} D_{1} \\
& +\frac{\left(1-\eta_{2} \beta\right) \tau_{1}+\left(1-\gamma+\eta_{1} \gamma\right)\left(\Delta-\rho_{1}\right)}{\left(\Delta-\rho_{1}\right)\left(\Delta-\tau_{2}\right)-\rho_{2} \tau_{1}} D_{2}+D_{3}, \\
0 & <L \\
& <\frac{\eta_{1}}{\eta_{2}-\left(\eta_{2}-\eta_{1}\right) \gamma}\left(\frac{\left(1-\eta_{2}\right)\left(\Delta-\tau_{2}\right)+\left(\eta_{1} \gamma+\eta_{2}-\eta_{2} \gamma\right) \rho_{2}}{\left(\Delta-\rho_{1}\right)\left(\Delta-\tau_{2}\right)-\rho_{2} \tau_{1}}\right. \\
& \left.\cdot D_{4}+\frac{\left(1-\eta_{2}\right) \tau_{1}+\left(\eta_{1} \gamma+\eta_{2}-\eta_{2} \gamma\right)\left(\Delta-\rho_{1}\right)}{\left(\Delta-\rho_{1}\right)\left(\Delta-\tau_{2}\right)-\rho_{2} \tau_{1}} D_{5}+D_{6}\right) .
\end{aligned}
$$

Theorem 7. Suppose that there exist positive constants $a, b$, and $d$ with $a<b<b / \Gamma \leq d$ such that the following conditions are fulfilled:

$$
\begin{aligned}
& \left(A_{1}\right) f(t, u) \leq d / \mu,(t, u) \in[0,1] \times[0, d], \\
& \left(A_{2}\right) f(t, u) \geq b / L,(t, u) \in\left[\eta_{1}, \eta_{2}\right] \times[b, b / \Gamma], \\
& \left(A_{3}\right) f(t, u) \leq a / \mu,(t, u) \in[0,1] \times[0, a] .
\end{aligned}
$$

Then the BVP (4) has at least three positive solutions $u_{1}, u_{2}, u_{3}$ satisfying $\left\|u_{i}\right\| \leq d(i=1,2,3)$ and

$$
\begin{aligned}
\min _{t \in\left[\eta_{1}, \eta_{2}\right]} u_{1}(t) & >b, \\
\left\|u_{2}\right\| & >a \text { with } \min _{t \in\left[\eta_{1}, \eta_{2}\right]} u_{2}(t)<b, \\
\left\|u_{3}\right\| & <a .
\end{aligned}
$$

Proof. For $u \in K$, we define

$$
\begin{aligned}
& \Phi(u)=\min _{t \in\left[\eta_{1}, \eta_{2}\right]} u(t), \\
& \varphi(u)=\Theta(u)=\Psi(u)=\|u\| .
\end{aligned}
$$


Then it is easy to know that $\Phi$ is a nonnegative continuous concave functional on $K$ and $\varphi, \Theta$ and $\Psi$ are nonnegative continuous convex functionals on $K$. In order to apply Theorem 1 to prove our main results, we use the operator $S$ and take $c=b / \Gamma$.

First, we assert that $S: \overline{K(\varphi, d)} \rightarrow \overline{K(\varphi, d)}$.

In fact, if $u \in \overline{K(\varphi, d)}$, then $0 \leq u(t) \leq d, t \in[0,1]$, which together with $\left(A_{1}\right)$ implies that

$$
\begin{aligned}
& \lambda_{i}[F u]=\frac{\gamma \rho_{i}}{\Delta} \int_{0}^{1} k\left(\eta_{1}, s\right) f(s, u(\alpha(s))) d s+\frac{\beta \tau_{i}}{\Delta} \\
& \cdot \int_{0}^{1} k\left(\eta_{2}, s\right) f(s, u(\alpha(s))) d s \\
& +\int_{0}^{1} \kappa_{i}(s) f(s, u(\alpha(s))) d s \\
& \leq\left(\frac{\gamma \rho_{i}}{\Delta} \int_{0}^{1} k\left(\eta_{1}, s\right) d s+\frac{\beta \tau_{i}}{\Delta} \int_{0}^{1} k\left(\eta_{2}, s\right) d s\right. \\
& \left.+\int_{0}^{1} \kappa_{i}(s) d s\right) \frac{d}{\mu}=\frac{D_{i} d}{\mu}, \quad i=1,2, \\
& \|F u\|=\max _{t \in[0,1]}(F u)(t) \leq \frac{\left(1-\eta_{2} \beta\right) \gamma}{\Delta} \\
& \cdot \int_{0}^{1} k\left(\eta_{1}, s\right) f(s, u(\alpha(s))) d s+\frac{\left(1-\gamma+\eta_{1} \gamma\right) \beta}{\Delta} \\
& \cdot \int_{0}^{1} k\left(\eta_{2}, s\right) f(s, u(\alpha(s))) d s+\frac{1}{2} \\
& \text { - } \int_{0}^{1}(1+s)(1-s)^{2} f(s, u(\alpha(s))) d s \\
& \leq\left(\frac{\left(1-\eta_{2} \beta\right) \gamma}{\Delta} \int_{0}^{1} k\left(\eta_{1}, s\right) d s\right. \\
& \left.+\frac{\left(1-\gamma+\eta_{1} \gamma\right) \beta}{\Delta} \int_{0}^{1} k\left(\eta_{2}, s\right) d s+\frac{5}{24}\right) \frac{d}{\mu} \\
& =\frac{D_{3} d}{\mu} .
\end{aligned}
$$

It follows from (70) that

$$
\begin{aligned}
& \varphi(S u)=\|S u\| \\
& \quad \leq \frac{\left(1-\eta_{2} \beta\right)\left(\Delta-\tau_{2}\right)+\left(1-\gamma+\eta_{1} \gamma\right) \rho_{2}}{\left(\Delta-\rho_{1}\right)\left(\Delta-\tau_{2}\right)-\rho_{2} \tau_{1}} \lambda_{1}[F u] \\
& +\frac{\left(1-\eta_{2} \beta\right) \tau_{1}+\left(1-\gamma+\eta_{1} \gamma\right)\left(\Delta-\rho_{1}\right)}{\left(\Delta-\rho_{1}\right)\left(\Delta-\tau_{2}\right)-\rho_{2} \tau_{1}} \lambda_{2}[F u] \\
& +\|F u\| \\
& \quad \leq\left(\frac{\left(1-\eta_{2} \beta\right)\left(\Delta-\tau_{2}\right)+\left(1-\gamma+\eta_{1} \gamma\right) \rho_{2}}{\left(\Delta-\rho_{1}\right)\left(\Delta-\tau_{2}\right)-\rho_{2} \tau_{1}} D_{1}\right. \\
& +\frac{\left(1-\eta_{2} \beta\right) \tau_{1}+\left(1-\gamma+\eta_{1} \gamma\right)\left(\Delta-\rho_{1}\right)}{\left(\Delta-\rho_{1}\right)\left(\Delta-\tau_{2}\right)-\rho_{2} \tau_{1}} D_{2} \\
& \left.+D_{3}\right) \frac{d}{\mu} \leq d .
\end{aligned}
$$

This shows that $S: \overline{K(\varphi, d)} \rightarrow \overline{K(\varphi, d)}$.
Next, we claim that $\{u \in K(\varphi, \Theta, \Phi, b, c, d): \Phi(u)>b\} \neq$ $\phi$ and $\Phi(S u)>b$ for $u \in K(\varphi, \Theta, \Phi, b, c, d)$.

Indeed, the constant function $(b+c) / 2 \in\{u \in$ $K(\varphi, \Theta, \Phi, b, c, d): \Phi(u)>b\}$. Moreover, if $u \in$ $K(\varphi, \Theta, \Phi, b, c, d)$, then $b \leq u(t) \leq c, t \in\left[\eta_{1}, \eta_{2}\right]$, which together with $\eta_{1} \leq t \leq \alpha(t) \leq \eta_{2}$ for $t \in\left[\eta_{1}, \eta_{2}\right]$ implies that $b \leq u(\alpha(t)) \leq c, t \in\left[\eta_{1}, \eta_{2}\right]$. In view of $\left(A_{2}\right)$, we have

$$
\begin{aligned}
& \lambda_{1}[F u]=\frac{\gamma \rho_{1}}{\Delta} \int_{0}^{1} k\left(\eta_{1}, s\right) f(s, u(\alpha(s))) d s+\frac{\beta \tau_{1}}{\Delta} \\
& \cdot \int_{0}^{1} k\left(\eta_{2}, s\right) f(s, u(\alpha(s))) d s \\
& +\int_{0}^{1} \kappa_{1}(s) f(s, u(\alpha(s))) d s \geq \frac{\gamma \rho_{1}}{\Delta} \\
& \cdot \int_{\eta_{1}}^{\eta_{2}} k\left(\eta_{1}, s\right) f(s, u(\alpha(s))) d s+\frac{\beta \tau_{1}}{\Delta} \\
& \cdot \int_{\eta_{1}}^{\eta_{2}} k\left(\eta_{2}, s\right) f(s, u(\alpha(s))) d s \\
& +\int_{\eta_{1}}^{\eta_{2}} \kappa_{1}(s) f(s, u(\alpha(s))) d s \\
& \geq\left(\frac{\gamma \rho_{1}}{\Delta} \int_{\eta_{1}}^{\eta_{2}} k\left(\eta_{1}, s\right) d s+\frac{\beta \tau_{1}}{\Delta} \int_{\eta_{1}}^{\eta_{2}} k\left(\eta_{2}, s\right) d s\right. \\
& \left.+\int_{\eta_{1}}^{\eta_{2}} \kappa_{1}(s) d s\right) \frac{b}{L}=\frac{D_{4} b}{L} \\
& \lambda_{2}[F u]=\frac{\gamma \rho_{2}}{\Delta} \int_{0}^{1} k\left(\eta_{1}, s\right) f(s, u(\alpha(s))) d s+\frac{\beta \tau_{2}}{\Delta} \\
& \cdot \int_{0}^{1} k\left(\eta_{2}, s\right) f(s, u(\alpha(s))) d s \\
& +\int_{0}^{1} \kappa_{2}(s) f(s, u(\alpha(s))) d s \geq \frac{\gamma \rho_{2}}{\Delta} \\
& \cdot \int_{\eta_{1}}^{\eta_{2}} k\left(\eta_{1}, s\right) f(s, u(\alpha(s))) d s+\frac{\beta \tau_{2}}{\Delta} \\
& \cdot \int_{\eta_{1}}^{\eta_{2}} k\left(\eta_{2}, s\right) f(s, u(\alpha(s))) d s \\
& +\int_{\eta_{1}}^{\eta_{2}} \kappa_{2}(s) f(s, u(\alpha(s))) d s \\
& \geq\left(\frac{\gamma \rho_{2}}{\Delta} \int_{\eta_{1}}^{\eta_{2}} k\left(\eta_{1}, s\right) d s+\frac{\beta \tau_{2}}{\Delta} \int_{\eta_{1}}^{\eta_{2}} k\left(\eta_{2}, s\right) d s\right. \\
& \left.+\int_{\eta_{1}}^{\eta_{2}} \kappa_{2}(s) d s\right) \frac{b}{L}=\frac{D_{5} b}{L} \\
& (F u)\left(\eta_{2}\right)=\frac{\gamma\left(1-\eta_{2}\right)}{\Delta} \int_{0}^{1} k\left(\eta_{1}, s\right) f(s, u(\alpha(s))) d s \\
& +\frac{1-\gamma+\eta_{1} \gamma}{\Delta} \int_{0}^{1} k\left(\eta_{2}, s\right) f(s, u(\alpha(s))) d s \\
& \geq \frac{\gamma\left(1-\eta_{2}\right)}{\Delta} \int_{\eta_{1}}^{\eta_{2}} k\left(\eta_{1}, s\right) f(s, u(\alpha(s))) d s
\end{aligned}
$$




$$
\begin{aligned}
& +\frac{1-\gamma+\eta_{1} \gamma}{\Delta} \int_{\eta_{1}}^{\eta_{2}} k\left(\eta_{2}, s\right) f(s, u(\alpha(s))) d s \\
& \geq\left(\frac{\gamma\left(1-\eta_{2}\right)}{\Delta} \int_{\eta_{1}}^{\eta_{2}} k\left(\eta_{1}, s\right) d s\right. \\
& \left.+\frac{1-\gamma+\eta_{1} \gamma}{\Delta} \int_{\eta_{1}}^{\eta_{2}} k\left(\eta_{2}, s\right) d s\right) \frac{b}{L}=\frac{D_{6} b}{L} .
\end{aligned}
$$

Since $S u$ is concave down on $[0,1]$, we have

$$
\frac{(S u)\left(\eta_{2}\right)-(S u)(0)}{\eta_{2}} \leq \frac{(S u)\left(\eta_{1}\right)-(S u)(0)}{\eta_{1}} .
$$

So,

$$
(S u)\left(\eta_{2}\right) \leq \frac{\eta_{2}}{\eta_{1}}(S u)\left(\eta_{1}\right)-\frac{\eta_{2}-\eta_{1}}{\eta_{1}}(S u)(0),
$$

which together with

$$
\begin{aligned}
(S u)(0)= & \gamma(S u)\left(\eta_{1}\right) \\
& +\frac{\Delta\left(\Delta-\tau_{2}\right) \lambda_{1}[F u]+\Delta \tau_{1} \lambda_{2}[F u]}{\left(\Delta-\rho_{1}\right)\left(\Delta-\tau_{2}\right)-\rho_{2} \tau_{1}}
\end{aligned}
$$

implies that

$$
(S u)\left(\eta_{1}\right) \geq \frac{\eta_{1}}{\eta_{2}-\left(\eta_{2}-\eta_{1}\right) \gamma}(S u)\left(\eta_{2}\right) .
$$

Therefore, it follows from (72) and (76) that

$$
\begin{aligned}
\Phi & (S u)=\min _{t \in\left[\eta_{1}, \eta_{2}\right]}(S u)(t)=\min \left\{(S u)\left(\eta_{1}\right),(S u)\left(\eta_{2}\right)\right\} \\
& \geq \min \left\{\frac{\eta_{1}}{\eta_{2}-\left(\eta_{2}-\eta_{1}\right) \gamma}(S u)\left(\eta_{2}\right),(S u)\left(\eta_{2}\right)\right\} \\
& =\frac{\eta_{1}}{\eta_{2}-\left(\eta_{2}-\eta_{1}\right) \gamma}(S u)\left(\eta_{2}\right) \\
& =\frac{\eta_{1}}{\eta_{2}-\left(\eta_{2}-\eta_{1}\right) \gamma}\left(\frac{\left(1-\eta_{2}\right)\left(\Delta-\tau_{2}\right)+\left(\eta_{1} \gamma+\eta_{2}-\eta_{2} \gamma\right) \rho_{2}}{\left(\Delta-\rho_{1}\right)\left(\Delta-\tau_{2}\right)-\rho_{2} \tau_{1}}\right. \\
& \cdot \lambda_{1}[F u]+\frac{\left(1-\eta_{2}\right) \tau_{1}+\left(\eta_{1} \gamma+\eta_{2}-\eta_{2} \gamma\right)\left(\Delta-\rho_{1}\right)}{\left(\Delta-\rho_{1}\right)\left(\Delta-\tau_{2}\right)-\rho_{2} \tau_{1}} \lambda_{2}[F u] \\
& \left.+(F u)\left(\eta_{2}\right)\right) \\
& \geq \frac{\eta_{1}}{\eta_{2}-\left(\eta_{2}-\eta_{1}\right) \gamma}\left(\frac{\left(1-\eta_{2}\right)\left(\Delta-\tau_{2}\right)+\left(\eta_{1} \gamma+\eta_{2}-\eta_{2} \gamma\right) \rho_{2}}{\left(\Delta-\rho_{1}\right)\left(\Delta-\tau_{2}\right)-\rho_{2} \tau_{1}}\right. \\
& \left.\cdot D_{4}+\frac{\left(1-\eta_{2}\right) \tau_{1}+\left(\eta_{1} \gamma+\eta_{2}-\eta_{2} \gamma\right)\left(\Delta-\rho_{1}\right)}{\left(\Delta-\rho_{1}\right)\left(\Delta-\tau_{2}\right)-\rho_{2} \tau_{1}} D_{5}+D_{6}\right) \frac{b}{L} \\
& >b .
\end{aligned}
$$

Thirdly, we assert that $\Phi(S u)>b$ for $u \in K(\varphi, \Phi, b, d)$ with $\Theta(S u)>c$.

To see this, we suppose $u \in K(\varphi, \Phi, b, d)$ and $\Theta(S u)=$ $\|S u\|>c$. Then

$$
\Phi(S u)=\min _{t \in\left[\eta_{1}, \eta_{2}\right]}(S u)(t) \geq \Gamma\|S u\|>\Gamma c=b .
$$

Finally, we prove that $\theta \notin R(\varphi, \Psi, a, d)$ and $\Psi(S u)<a$ for $u \in R(\varphi, \Psi, a, d)$ with $\Psi(u)=a$.

Indeed, it follows from $\Psi(\theta)=0<a$ that $\theta \notin$ $R(\varphi, \Psi, a, d)$. Moreover, if $u \in R(\varphi, \Psi, a, d)$ and $\Psi(u)=a$, then $0 \leq u(t) \leq a, t \in[0,1]$, which together with $\left(A_{3}\right)$ implies that

$$
\begin{aligned}
& \lambda_{i}[F u]=\frac{\gamma \rho_{i}}{\Delta} \int_{0}^{1} k\left(\eta_{1}, s\right) f(s, u(\alpha(s))) d s+\frac{\beta \tau_{i}}{\Delta} \\
& \cdot \int_{0}^{1} k\left(\eta_{2}, s\right) f(s, u(\alpha(s))) d s \\
& +\int_{0}^{1} \kappa_{i}(s) f(s, u(\alpha(s))) d s \\
& \leq\left(\frac{\gamma \rho_{i}}{\Delta} \int_{0}^{1} k\left(\eta_{1}, s\right) d s+\frac{\beta \tau_{i}}{\Delta} \int_{0}^{1} k\left(\eta_{2}, s\right) d s\right. \\
& \left.+\int_{0}^{1} \kappa_{i}(s) d s\right) \frac{a}{\mu}=\frac{D_{i} a}{\mu}, \quad i=1,2, \\
& \|F u\|=\max _{t \in[0,1]}(F u)(t) \leq \frac{\left(1-\eta_{2} \beta\right) \gamma}{\Delta} \\
& \cdot \int_{0}^{1} k\left(\eta_{1}, s\right) f(s, u(\alpha(s))) d s+\frac{\left(1-\gamma+\eta_{1} \gamma\right) \beta}{\Delta} \\
& \cdot \int_{0}^{1} k\left(\eta_{2}, s\right) f(s, u(\alpha(s))) d s+\frac{1}{2} \\
& \cdot \int_{0}^{1}(1+s)(1-s)^{2} f(s, u(\alpha(s))) d s \\
& \leq\left(\frac{\left(1-\eta_{2} \beta\right) \gamma}{\Delta} \int_{0}^{1} k\left(\eta_{1}, s\right) d s\right. \\
& \left.+\frac{\left(1-\gamma+\eta_{1} \gamma\right) \beta}{\Delta} \int_{0}^{1} k\left(\eta_{2}, s\right) d s+\frac{5}{24}\right) \frac{a}{\mu}=\frac{D_{3} a}{\mu} .
\end{aligned}
$$

In view of (79), we have

$$
\begin{aligned}
& \Psi(S u)=\|S u\| \\
& \quad \leq \frac{\left(1-\eta_{2} \beta\right)\left(\Delta-\tau_{2}\right)+\left(1-\gamma+\eta_{1} \gamma\right) \rho_{2}}{\left(\Delta-\rho_{1}\right)\left(\Delta-\tau_{2}\right)-\rho_{2} \tau_{1}} \lambda_{1}[F u] \\
& +\frac{\left(1-\eta_{2} \beta\right) \tau_{1}+\left(1-\gamma+\eta_{1} \gamma\right)\left(\Delta-\rho_{1}\right)}{\left(\Delta-\rho_{1}\right)\left(\Delta-\tau_{2}\right)-\rho_{2} \tau_{1}} \lambda_{2}[F u] \\
& +\|F u\| \\
& \quad \leq\left(\frac{\left(1-\eta_{2} \beta\right)\left(\Delta-\tau_{2}\right)+\left(1-\gamma+\eta_{1} \gamma\right) \rho_{2}}{\left(\Delta-\rho_{1}\right)\left(\Delta-\tau_{2}\right)-\rho_{2} \tau_{1}} D_{1}\right. \\
& \left.+\frac{\left(1-\eta_{2} \beta\right) \tau_{1}+\left(1-\gamma+\eta_{1} \gamma\right)\left(\Delta-\rho_{1}\right)}{\left(\Delta-\rho_{1}\right)\left(\Delta-\tau_{2}\right)-\rho_{2} \tau_{1}} D_{2}+D_{3}\right) \\
& \quad \frac{a}{\mu}<a,
\end{aligned}
$$

as required. 
To sum up, all the hypotheses of Theorem 1 are satisfied. Hence, the BVP (4) has at least three positive solutions $u_{1}, u_{2}$, $u_{3}$ satisfying $\left\|u_{i}\right\| \leq d(i=1,2,3)$ and

$$
\begin{aligned}
\min _{t \in\left[\eta_{1}, \eta_{2}\right]} u_{1}(t) & >b, \\
\left\|u_{2}\right\| & >a \text { with } \min _{t \in\left[\eta_{1}, \eta_{2}\right]} u_{2}(t)<b, \\
\left\|u_{3}\right\| & <a .
\end{aligned}
$$

\section{An Example}

Example 1. Consider the following BVP:

$$
\begin{aligned}
& u^{\prime \prime \prime}(t)+f(t, u(\alpha(t)))=0, \quad t \in(0,1), \\
& u(0)=\frac{1}{2} u\left(\frac{1}{4}\right)+\int_{0}^{1} u(t) d\left(\frac{3}{2} t^{2}-t\right), \\
& u^{\prime \prime}(0)=0, \\
& u(1)=\frac{1}{4} u\left(\frac{1}{2}\right)+\int_{0}^{1} u(t) d\left(\frac{1}{4} t^{2}\right),
\end{aligned}
$$

where

$$
\begin{aligned}
f(t, u) & = \begin{cases}20 u^{2}+\left(\frac{1}{20}-u\right) t(1-t), & (t, u) \in[0,1] \times\left[0, \frac{1}{20}\right], \\
\frac{1}{20}+1980\left(u-\frac{1}{20}\right)^{2}+\left(u-\frac{1}{20}\right)\left(\frac{1}{10}-u\right) t(1-t), & (t, u) \in[0,1] \times\left(\frac{1}{20}, \frac{1}{10}\right), \\
5+\frac{1}{10}\left(u-\frac{1}{10}\right)^{2} t(1-t), & (t, u) \in[0,1] \times\left[\frac{1}{10},+\infty\right),\end{cases} \\
\alpha(t) & = \begin{cases}\frac{\sqrt{2 t}}{2}, & t \in\left[0, \frac{1}{2}\right], \\
\frac{\sqrt{2 t-1}+1}{2}, & t \in\left(\frac{1}{2}, 1\right] .\end{cases}
\end{aligned}
$$

Since $\Lambda_{1}(t)=(3 / 2) t^{2}-t$ and $\Lambda_{2}(t)=(1 / 4) t^{2}, t \in[0,1]$, a simple calculation shows that

$$
\begin{aligned}
\int_{0}^{1} d \Lambda_{1}(t) & =\int_{0}^{1} t d \Lambda_{1}(t)=\frac{1}{2}, \\
\int_{0}^{1} d \Lambda_{2}(t) & =\frac{1}{4} \\
\int_{0}^{1} t d \Lambda_{2}(t) & =\frac{1}{6} \\
\kappa_{1}(s) & =\frac{1}{8} s^{4}-\frac{1}{6} s^{3}+\frac{1}{24} \\
\kappa_{2}(s) & =\frac{1}{48}\left(1-s^{2}\right)^{2},
\end{aligned}
$$$$
s \in[0,1] .
$$

At the same time, in view of $\eta_{1}=\beta=1 / 4$ and $\eta_{2}=\gamma=1 / 2$, we get

$$
\begin{aligned}
\Delta & =\frac{17}{32}, \\
\rho_{1} & =\frac{1}{16}, \\
\tau_{1} & =\frac{5}{16}, \\
\rho_{2} & =\frac{3}{32},
\end{aligned}
$$

$$
\begin{aligned}
\tau_{2} & =\frac{11}{96}, \\
\Gamma & =\frac{2}{5}, \\
D_{1} & =\frac{397}{10880}, \\
D_{2} & =\frac{3511}{195840}, \\
D_{3} & =\frac{845}{3264}, \\
D_{4} & =\frac{26561}{2088960}, \\
D_{5} & =\frac{25487}{4177920}, \\
D_{6} & =\frac{13}{408} .
\end{aligned}
$$

If we choose $\mu=1, L=1 / 50, a=1 / 20, b=1 / 10$, and $d=6$, then all the conditions of Theorem 7 are fulfilled. Therefore, it follows from Theorem 7 that the BVP (82) has at least three positive solutions $u_{1}, u_{2}, u_{3}$ satisfying $\left\|u_{i}\right\| \leq 6(i=1,2,3)$ and

$$
\begin{aligned}
\min _{t \in[1 / 4,1 / 2]} u_{1}(t) & >\frac{1}{10}, \\
\left\|u_{2}\right\| & >\frac{1}{20} \quad \text { with } \min _{t \in[1 / 4,1 / 2]} u_{2}(t)<\frac{1}{10}, \\
\left\|u_{3}\right\| & <\frac{1}{20} .
\end{aligned}
$$




\section{Competing Interests}

The authors declare that they have no competing interests.

\section{References}

[1] M. Greguš, Third Order Linear Differential Equations, vol. 22, Reidel, Dordrecht, The Netherlands, 1987.

[2] M. Abushammala, S. A. Khuri, and A. Sayfy, "A novel fixed point iteration method for the solution of third order boundary value problems," Applied Mathematics and Computation, vol. 271, pp. 131-141, 2015.

[3] D. R. Anderson, "Green's function for a third-order generalized right focal problem," Journal of Mathematical Analysis and Applications, vol. 288, no. 1, pp. 1-14, 2003.

[4] Z. Bai and X. Fei, "Existence of triple positive solutions for a third order generalized right focal problem," Mathematical Inequalities and Applications, vol. 9, no. 3, pp. 437-444, 2006.

[5] X. Feng, H. Feng, and D. Bai, "Eigenvalue for a singular thirdorder three-point boundary value problem," Applied Mathematics and Computation, vol. 219, no. 18, pp. 9783-9790, 2013.

[6] J. R. Graef and L. Kong, "Positive solutions for third order semipositone boundary value problems," Applied Mathematics Letters, vol. 22, no. 8, pp. 1154-1160, 2009.

[7] J. R. Graef and J. R. L. Webb, "Third order boundary value problems with nonlocal boundary conditions," Nonlinear Analysis: Theory, Methods \& Applications, vol. 71, no. 5-6, pp. 1542-1551, 2009.

[8] J. R. Graef and B. Yang, "Positive solutions of a third order nonlocal boundary value problem," Discrete and Continuous Dynamical Systems: Series S, vol. 1, no. 1, pp. 89-97, 2008.

[9] L.-J. Guo, J.-P. Sun, and Y.-H. Zhao, "Existence of positive solutions for nonlinear third-order three-point boundary value problems," Nonlinear Analysis: Theory, Methods \& Applications, vol. 68 , no. 10, pp. 3151-3158, 2008.

[10] B.-W. Niu, J.-P. Sun, and Q.-Y. Ren, “Two positive solutions of third-order BVP with integral boundary condition and signchanging Green's function," Journal of Function Spaces, vol. 2015, Article ID 491423, 8 pages, 2015.

[11] A. P. Palamides and G. Smyrlis, "Positive solutions to a singular third-order three-point boundary value problem with an indefinitely signed Green's function," Nonlinear Analysis: Theory, Methods \& Applications, vol. 68, no. 7, pp. 2104-2118, 2008.

[12] J.-P. Sun and H.-B. Li, "Monotone positive solution of nonlinear third-order BVP with integral boundary conditions," Boundary Value Problems, vol. 2010, Article ID 874959, 12 pages, 2010.

[13] J.-P. Sun and J. Zhao, "Multiple positive solutions for a thirdorder three-point BVP with sign-changing Green's function," Electronic Journal of Differential Equations, vol. 2012, no. 118, pp. $1-7,2012$.

[14] J.-P. Sun and J. Zhao, "Iterative technique for a third-order three-point BVP with sign-changing Green's function," Electronic Journal of Differential Equations, vol. 2013, no. 215, pp. 1-9, 2013.

[15] Y. Sun, "Positive solutions of singular third-order three-point boundary value problem," Journal of Mathematical Analysis and Applications, vol. 306, no. 2, pp. 589-603, 2005.

[16] Y. Sun, "Positive solutions for third-order three-point nonhomogeneous boundary value problems," Applied Mathematics Letters, vol. 22, no. 1, pp. 45-51, 2009.
[17] F. J. Torres, "Positive solutions for a third-order three-point boundary-value problem," Electronic Journal of Differential Equations, vol. 2013, no. 147, pp. 1-11, 2013.

[18] Y. Wang and W. Ge, "Existence of solutions for a third order differential equation with integral boundary conditions," Computers and Mathematics with Applications, vol. 53, no. 1, pp. 144154, 2007.

[19] Z. L. Wei, "Some necessary and sufficient conditions for existence of positive solutions for third order singular sublinear multi-point boundary value problems," Acta Mathematica Scientia, vol. 34, no. 6, pp. 1795-1810, 2014.

[20] Q. Yao, "Positive solutions of singular third-order three-point boundary value problems," Journal of Mathematical Analysis and Applications, vol. 354, no. 1, pp. 207-212, 2009.

[21] T. Jankowski, "Existence of positive solutions to third order differential equations with advanced arguments and nonlocal boundary conditions," Nonlinear Analysis: Theory, Methods \& Applications, vol. 75, no. 2, pp. 913-923, 2012.

[22] J. R. L. Webb and G. Infante, "Positive solutions of nonlocal boundary value problems: a unified approach," Journal of the London Mathematical Society, vol. 74, no. 3, pp. 673-693, 2006.

[23] J. R. L. Webb and G. Infante, "Positive solutions of nonlocal boundary value problems involving integral conditions," NoDEA: Nonlinear Differential Equations and Applications, vol. 15, no. 1-2, pp. 45-67, 2008.

[24] G. Infante, P. Pietramala, and M. Zima, "Positive solutions for a class of nonlocal impulsive BVPs via fixed point index," Topological Methods in Nonlinear Analysis, vol. 36, no. 2, pp. 263-284, 2010.

[25] T. Jankowski, "Positive solutions for second order impulsive differential equations involving Stieltjes integral conditions," Nonlinear Analysis: Theory, Methods \& Applications, vol. 74, no. 11, pp. 3775-3785, 2011.

[26] J.-P. Sun, P. Yan, Y.-H. Zhao, and F.-D. Kong, "Existence of positive solution for a third-order BVP with advanced arguments and Stieltjes integral boundary conditions," Mathematical Problems in Engineering, vol. 2013, Article ID 157849, 9 pages, 2013.

[27] J.-P. Sun, P. Yan, and F.-D. Kong, "Third-order BVP with advanced arguments and Stieltjes integral boundary conditions," Applied Mathematics E-Notes, vol. 13, pp. 160-173, 2013.

[28] D. J. Guo and V. Lakshmikantham, Nonlinear Problems in Abstract Cones, vol. 5 of Notes and Reports in Mathematics in Science and Engineering, Academic Press, Boston, Mass, USA, 1988.

[29] M. A. Krasnoselskii, Positive Solutions of Operator Equations, Noordhoff, Groningen, The Netherlands, 1964.

[30] R. I. Avery and A. C. Peterson, "Three positive fixed points of nonlinear operators on ordered Banach spaces," Computers \& Mathematics with Applications, vol. 42, no. 3-5, pp. 313-322, 2001. 


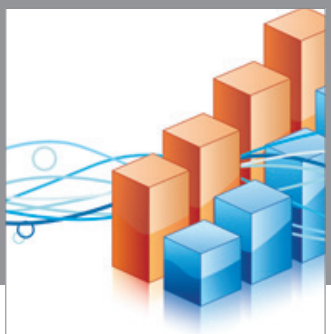

Advances in

Operations Research

vatem alat4

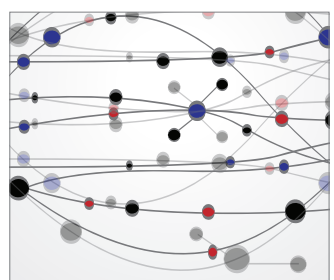

\section{The Scientific} World Journal
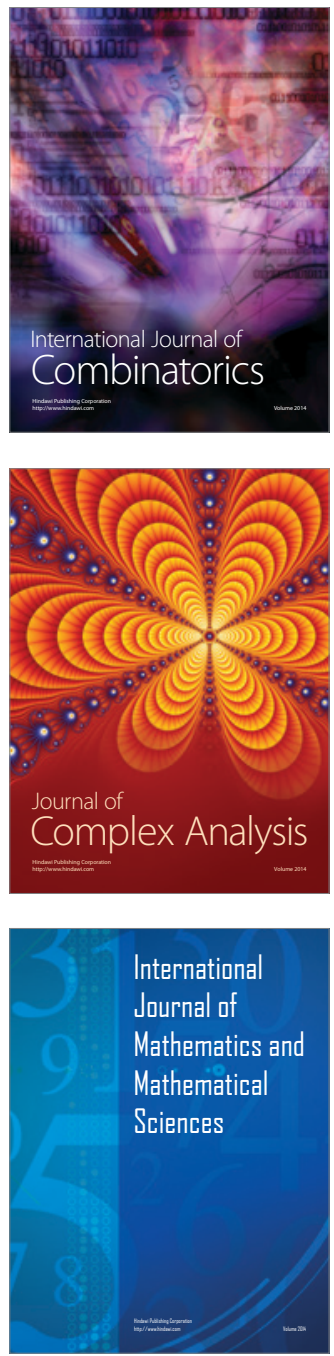
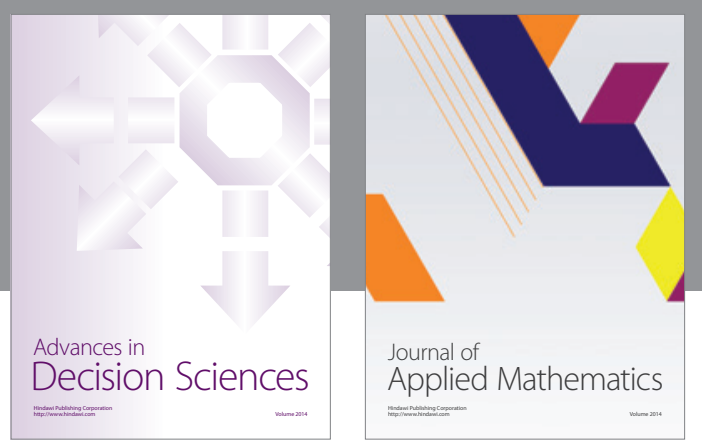

Algebra

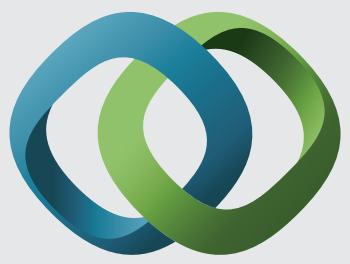

\section{Hindawi}

Submit your manuscripts at

http://www.hindawi.com
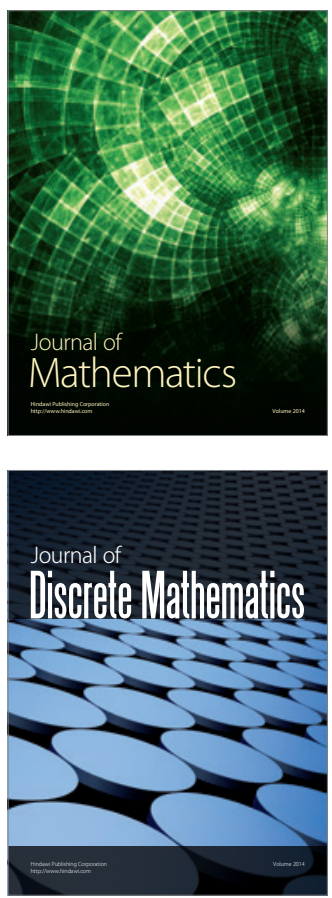

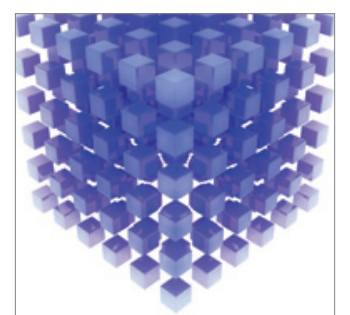

Mathematical Problems in Engineering
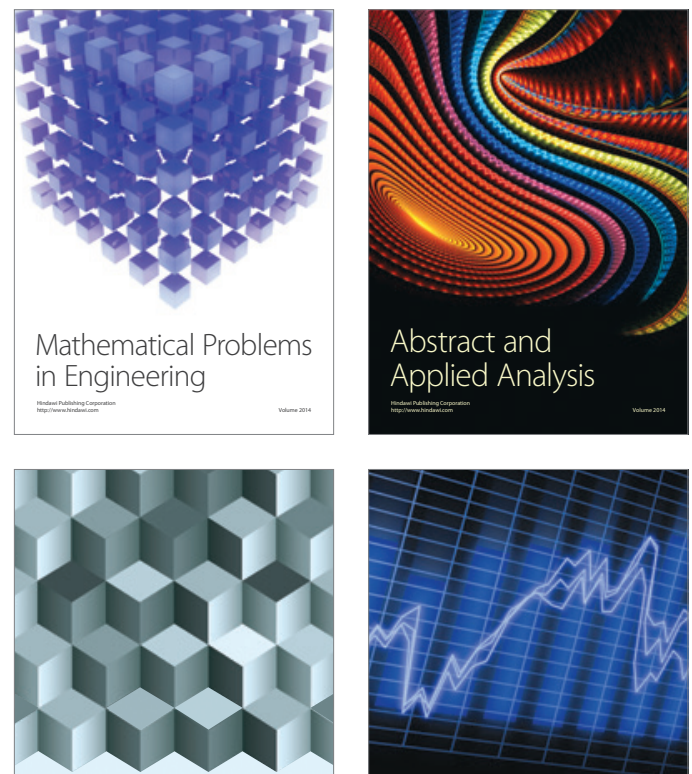

Journal of

Function Spaces

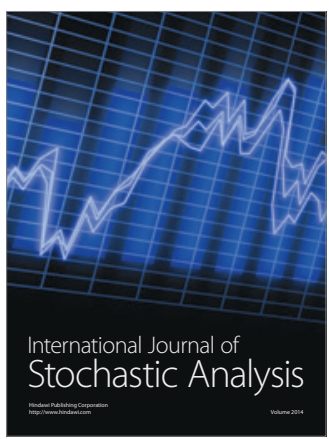

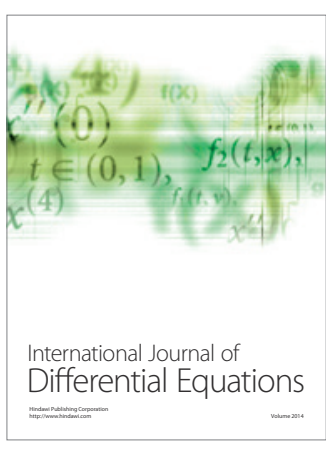
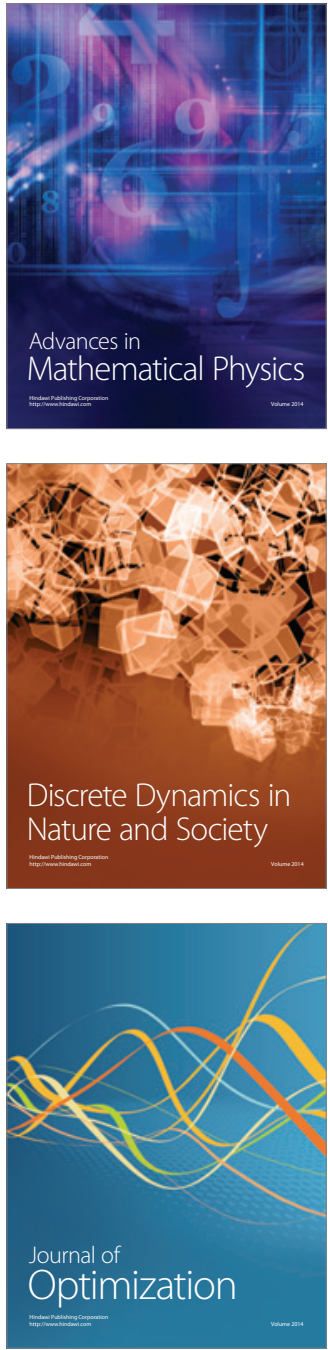